\title{
Turbulent Entrainment at the Boundaries of the Convective Cores of Main-sequence Stars
}

\author{
E. I. Staritsin* \\ K.A. Barkhatova Kourovka Astronomical Observatory, \\ B.N. El'tsin Ural Federal University, \\ Ekaterinburg, Russia \\ Received August 15, 2012; in final form, October 31, 2012
}

\begin{abstract}
Extra mixing of matter in stellar interiors at the boundaries of the convective cores of mainsequence stars is considered for the first time using the physical model of turbulent entrainment developed by Arnett and collaborators based on three-dimensional hydrodynamical simulations. The model takes into account the energy that goes into mixing the matter of the convective core and layers stable against convection located above the core. It is shown that the extent of the region of extra mixing expressed in units of the pressure scale height is not constant, and decreases as the star evolves along the main sequence. Adequate allowance for extra mixing at the boundaries of convective cores is necessary to clarify the relative importance of different mixing mechanisms in stellar interiors, as well as to determine stellar parameters using asteroseismlogy.
\end{abstract}

DOI: $10.1134 / \mathrm{S} 1063772913050089$

\section{INTRODUCTION}

It is well known that one of the most important characteristics of a star-its luminosity-is determined by the stellar mass and chemical composition. The chemical composition of a star varies due to nuclear reactions occurring in the stellar interior. In the main-sequence stage, hydrogen is converted into helium in the central regions of the star. The increase in the helium abundance decreases the opacity of the stellar matter, which, in turn, increases the stellar luminosity. The total increase in the stellar luminosity during the main-sequence lifetime is determined by the total mass of hydrogen converted into helium. In standard models for stellar evolution, this mass is determined by the dimensions of the convective region in the stellar interior. As was shown by the earliest large-scale computations of the stellar evolution on the main sequence, the calculated increase in the stellar luminosity is lower than the observed increase for stars in open clusters [1-3].

The motion of matter in the zone of convective instability has a turbulent character. A turbulent flow moving upward spreads in the horizontal plane in the vicinity of the boundary of the turbulent region and then descends, flowing around the upward-moving stream. In the region of horizontal flow, the velocities of the different horizontal layers varies in the vertical direction. This shear flow catches up superposed

${ }^{*}$ E-mail: Eugenij.Staritsin@usu.ru layers stable to convection into the motion, bringing their matter into the turbulent region.

In the stellar interior, the matter of layers that are unstable to convection located in central and close to central regions of the star is enriched in the products of nuclear fusion, is well mixed, and differs from the matter of the superposed layers in its higher molar mass. Therefore, mixing of matter in the regions of convective turbulence and matter in the superposed stable regions increases the gravitational potential energy of these layers, which is possible only due to the kinetic energy in the shear turbulent flow close to the boundary between the convective, turbulent region and stable regions. This boundary moves along the stable layers of matter as a result of the continual involvement of new layers in the turbulent motion. This increases the mass of hydrogen available for nuclear fusion, thereby facilitating a larger increase in the stellar luminosity on the main sequence. The velocity of the boundary and the distance it moves are determined by the ratio of the excess kinetic energy in the shear turbulent flow close to the boundary and the variation of the potential energy resulting from the involvement of new layers in the turbulent motion. The growth of the extent of the stable layers involved in the turbulent motion must balance the growth of the potential energy associated with the equalization of the chemical compositions of these and underlaying layers. Since the kinetic energy of the turbulent motion that can go into increasing the 
potential energy is limited, the extent of the layers that can be involved in the turbulent motion is also limited.

There is no theoretical model for the well known qualitative picture of turbulent entrainment described above. Therefore, the possible inclusion of stable, circum-boundary regions into the zone of convective turbulence has long been described using the parameter $\alpha: d=\alpha H_{P}$, where $d$ is the distance between the boundaries of the regions of convective instability and convective turbulence and $H_{P}$ is pressure scale height. For example, for achieve agreement between the computed and observed increase of the stellar luminosity on the main sequence invoking turbulent entrainment alone, the parameter $\alpha$ must be $\sim(0.15-0.17)[1]$.

Another possible way to study the properties of stellar cores is analysis of the gravitational modes of the pulsations of variable stars. The most interesting objects for this purpose are main-sequence B stars. The frequency spectrum of the pulsations of these stars does not contain solar-type oscillations, and instead contains $\mathrm{p}$ and $\mathrm{g}$ modes of pulsations exited by the $\kappa$ mechanism. The identification of the frequency spectrum and determination of the degree and azimuthal number of the mode using multicolor photometry and spectroscopy are simplest for these stars. Seismological parameters of several main-sequence $\mathrm{B}$ stars have been determined by fitting the computed and observed frequency spectra (Table 1). The parameter $\alpha$ can vary over a fairly broad range, both for a particular star and for an ensemble of stars. The observed frequency spectra for HD 180642, 12 Lac, and $\nu$ Eri can also be obtained numerically for $\alpha=0$. For the rest of the listed stars, $\alpha>0$. The largest value of $\alpha$ was found for the close binary $\theta$ Oph.

The progress of computational hydrodynamics over the last several decades has enabled direct numerical simulations of complex hydrodynamic processes under astrophysical conditions. Threedimensional numerical modeling of the flow at the boundary between the zone of convective turbulence and the overlying stable zones has confirmed the existence of turbulent entrainment in stellar interiors [13]. Computations were made for part of the convective core and the adjacent superposed stellar layer for a $23 M_{\odot}$ star with a hydrogen-burning core and for a convective, carbon-burning shell with stable underlying and overlying layers for the same mass star. The extent of the computational domain in the vertical direction was $\sim 5 H_{P}$ in the former case and $\sim 3 H_{P}$ in the latter case. The dimensions of the computational domain in the two other directions exceeded the size of the largest turbulent vortices.

The time integrations are limited by the time for a sound wave to cross the computational domain. The velocity of the convective motions in the core is relatively low; for this reason, the luminosity at the lower boundary of the computational domain was artificially increased by an order of magnitude. This made it possible to cover five typical turbulence turnover times. The computations for the carbon-burning shell covered eight typical turbulence turnover times. In each case, a solution that can be considered to represent an equilibrium in a statistical sense was achieved in a time that was shorter than the convective turnover time. These numerical results made it possible, for the first time, to formulate a model for turbulent entrainment at the boundaries of convective zones in stellar interiors that can be applied to traditional one-dimensional (1D) stellar-evolution computations [13-15].

In the present study, we investigate the mixing of matter at the boundary of the convective zone in the central region of a star using the turbulententrainment model developed in [13-15].

\section{BASIC EQUATIONS}

The velocity $V_{e}$ of the penetration of the convective-turbulence boundary into the stable layers is determined by the turbulent-entrainment law

$$
V_{e} / V_{t}=A \mathrm{Ri}_{B}^{-n},
$$

where the bulk Richardson number $\mathrm{Ri}_{B}$ has the form

$$
\mathrm{Ri}_{B}=\frac{\triangle b \ell}{V_{t}^{2}},
$$

$V_{t}$ is the typical value of turbulent velocity pulsations, and $\ell$ is the largest scale for the turbulence. If the excess kinetic energy in the turbulent flow that is responsible for increasing the potential energy of the mixed stellar layers is determined in the immediate vicinity of the boundary of the region of convective turbulence, the power $n$ in the turbulent-entrainment law should be unity [16].

According to Meakin and Arnett [13], the chemically homogeneous zone of convective turbulence and neighboring stellar layers are separated by a boundary layer with a thickness $h \sim 2 V_{c} / N$, where $V_{c}$ is the convective velocity and $N$ is the buoyancy frequency, which is given by the expression

$$
N^{2}=\frac{g \delta}{H_{P}}\left(\nabla_{a d}-\nabla+\nabla_{\mu} / \delta\right),
$$

where $g$ is the free-fall acceleration, $\nabla$ the temperature gradient, $\nabla_{a d}$ the adiabatic temperature gradient, $\nabla_{\mu}$ the gradient of the mean molar mass, $\delta=$ $(4-3 \beta) / \beta$, and $\beta$ is the ratio of the radiation pressure to the total pressure. The buoyancy jump $\triangle b$ is 
Table 1. Seismological parameters of stars

\begin{tabular}{l|l|c|c|c|c}
\hline \multicolumn{1}{c|}{ Star } & Spectrum & $M, M_{\odot}$ & $X_{c}$ & $\alpha$ & Ref. \\
\hline HD 46202 & O9V & $\sim 24$ & $\sim 0.58$ & $0.05-0.15$ & {$[4]$} \\
$\beta$ CMa & B1II-III & $13.0-14.2$ & $0.11-0.13$ & $0.15-0.25$ & {$[5]$} \\
HD180642 & B1.5II-III & $11.4-11.8$ & $0.21-0.25$ & $0.00-0.05$ & {$[6]$} \\
$\delta$ Cet & B2IV & $10.0-10.4$ & $\sim 0.25$ & $0.15-0.25$ & {$[7]$} \\
$\theta$ Oph & B2IV & $7.9-8.5$ & $0.36-0.40$ & $0.37-0.51$ & {$[8]$} \\
12 Lac & B2III & $10.0-14.4$ & $0.13-0.21$ & $0.00-0.40$ & {$[9]$} \\
$\nu$ Eri & B2II & $9.0-9.9$ & $0.24-0.27$ & $0.00-0.10$ & {$[10]$} \\
HD 129929 & B3V & $\sim 9.35$ & $\sim 0.35$ & $0.05-0.15$ & {$[11]$} \\
HD 50230 & B3V & $\sim 7.0$ & $\sim 0.28$ & $0.20-0.30$ & {$[12]$} \\
\hline
\end{tabular}

determined by integrating over a distance equal to the thickness of the boundary layer:

$$
\triangle b=\int_{h} N^{2} d r .
$$

The values of the variables in the turbulent-entrainment law $V_{e}, V_{t}, \triangle b$, and $\ell$ were found via threedimensional (3D) numerical simulations of the flow hydrodynamics in the vicinity of the boundary layer of the convective core in the central hydrogen-burning stage, and in the vicinity of the lower and upper boundary layers of the convective, carbon-burning shell. Using these values, Meakin and Arnett [13] found the parameters of the turbulent-entrainment law $n=1.05 \pm 0.21$ and $\log A=0.027 \pm 0.38$.

Analysis of the numerical data on the dynamics of the motion in the turbulent convection zone obtained in [13] led to the conclusion [15] that the rate of dissipation of the kinetic energy of the turbulent motions in the convective-turbulence zones in stellar interiors $\epsilon_{k}$ can be described by the well-known Kolmogorov relation [17]:

$$
\epsilon_{k}=\frac{V_{t}^{3}}{\ell}
$$

A method for computing the rate of dissipation of the kinetic energy based on data from 1D stellar models, without 3D hydrodynamic simulations, was proposed in [14]. The rate of dissipation of the kinetic energy is related to the specific kinetic energy $E_{k, i s o}$, which is determined from the isotropic component of the turbulent-flow velocity:

$$
\epsilon_{k}=\frac{\left(2 E_{k, i s o}\right)^{2 / 3}}{H_{P}} .
$$

If the average rate of the entropy variation $\langle\dot{s}\rangle_{m}$ is defined as

$$
\langle\dot{s}\rangle_{m}=\frac{\left\langle\epsilon_{n}+\epsilon_{k}\right\rangle_{m}}{\langle T\rangle_{m}}
$$

where the averaging is performed over the mass of gas $m$ supposed to be involved in the convective turbulence, $\epsilon_{n}$ is the rate of nuclear energy generation, $T$ is the temperature, and averaged values are shown in angular brackets $\langle\ldots\rangle$, the convective luminosity $L_{c}$ can be found from the formula:

$$
L_{c}(\tilde{m})=\xi \int_{\tilde{m}}\left(\epsilon_{n}+\epsilon_{k}-T\langle\dot{s}\rangle_{m}\right) d m^{\prime},
$$

and the flux of kinetic energy in the turbulent region $L_{k}$ from the formula:

$$
L_{k}(\tilde{m})=\int_{\tilde{m}}\left(L_{c} \nabla a d \frac{d r^{\prime}}{H_{P}}-\epsilon_{k} d m^{\prime}\right) .
$$

The isotropic component of the specific kinetic energy of the turbulent flow $E_{k, i s o}$ is approximately constant over the region of convective turbulence. The value of $E_{k, i s o}$ can be found by requiring the kinetic-energy flux to vanish at the boundary of the convective turbulence, $L_{k}(m)=0$.

The largest turbulence scale $\ell$ in (2) is equal to $\ell_{\mathrm{Ri}} \sim 0.15 H_{P}-0.18 H_{P}$ for the convective core and the upper boundary of the convective shell [13], while $\ell_{\epsilon} \sim H_{P}$ in (4) for the convective shell [15]. Thus, the values of $\ell$ in (2) and (4) may differ.

Knowing the stellar structure, it is possible to determine the buoyancy jump $\triangle b$ at the boundary of the region of convective instability (3) and the rate of dissipation of the kinetic energy $\epsilon_{k}$ [see (5)-(8)]. Specifying $\ell$ in (2) and (4), it is possible to find the rate of penetration of the boundary of the region of 
convective turbulence $V_{e}$ using (1), (2), and (4). The distance $d$ over which this boundary shifts during an evolutionary time step $\Delta t$ is given by

$$
d=V_{e} \triangle t .
$$

After determining the position of the boundary of the region of convective turbulence, the chemical composition inside this region is equated to a homogeneous composition.

In our present study, the mixing of matter at the boundary of the convective cores of 16 and $24 M_{\odot}$ main-sequence stars is investigated using the turbulent-entrainment model described above. The computations were carried out using the code described in [18], with the modifications [19]. The initial stellar chemical composition was specified to be close to the solar composition: $(X, Z)=(0.70,0.02)$, where $X$ and $Z$ are the mass abundances of hydrogen and heavy elements. The opacity tables [20] were used for high-temperature regions $(\log T \geq 4.0)$, and the opacity tables [21] for low-temperature regions $(\log T<4.0)$. The velocity of the boundary of the region of convective turbulence in the overlying layers $V_{e}$ is determined by the turbulent entrainment law (1), and the parameter $n$ was specified to be equal to the value derived in [13] for physical conditions typical for stellar interiors. This value of $n$ is essentially equal to unity in our case, as should be the case based on the condition of energy balance [16]. The value of $A$ can be very different for laboratory and geophysical turbulent entrainment [22]. The velocity of the convective turbulence boundary motion is also determined by the largest turbulence scale. Assuming that the values of $\ell$ in (2) and (4) are different, after substituting (2) and (4) into (1), we obtain in the expression for the velocity the factor $\ell_{\epsilon}^{(2 n+1) / 3} / \ell_{\mathrm{Ri}}^{n}$. In the present paper, the value of $\ell$ is specified to be $0.10 H_{P}$ in both (2) and (4). Any inaccuracy in $V_{e}$ due to this choice of $\ell$ is compensated by the parameter $A$, which is fitted so as to satisfy asteroseismology data.

\section{RESULTS OF THE COMPUTATIONS}

Formula (7) for computing the convective luminosity for the case of a carbon-burning convective shell was suggested in [14]. Radiative transfer is inefficient in such a shell, and $\xi=1$. Radiative transfer plays an appreciable role in energy transport in the convective core in the central hydrogen-burning stage of a massive star. For this reason, we added to (7) the factor

$$
\xi=\frac{1}{m} \int_{0}^{m}\left(1-\frac{\nabla a d}{\nabla}\right) d m^{\prime},
$$

where $m$ is the mass of the region unstable to convection and the integrand is the ratio of the convective and total luminosities (according to the mixinglength theory [23]) as a function of the Lagrangian variable $m^{\prime}$. The convective luminosities in the cores of 16 and $24 M_{\odot}$ stars computed using (7) differ from the luminosities computed according to the mixinglength theory by less than $20 \%$, independent of the hydrogen abundance in the convective core (Fig. 1). The mixing-length theory is a simplified crude model for the convection intended for use in computing the convective energy flux [23]. In this model, the real flux of matter in the convective region of the star is replaced by radial motions of identical convective elements. Computation of the convective energy flux requires assumptions about the values of several parameters: the fraction of the work done by the buoyancy force that goes into accelerating the convective element, the distance traveled by the element before it loses its identity, the correlation coefficient for fluctuations of the temperature and velocity, and some others. The computed average values of these parameters differ from the typical values adopted in mixinglength theory by tens of percent, and may depend on the depth of the convective zone [13]. Therefore, the convective energy flux provided by the mixing-length theory is not completely accurate.

Substituting (1), (2), and (4) into (9) yields an expression for the dependence of the distance $d$ between the boundaries of the regions of convective instability and convective turbulence on the hydrogen abundance in the region of convective turbulence $X_{c}$ :

$$
d\left(X_{c}\right)=A \frac{V_{t}^{2 n+1}\left(X_{c}\right)}{\ell^{n}} \frac{\triangle t}{\left[\triangle b\left(X_{c}, \triangle t\right)\right]^{n}} .
$$

The formation of the buoyancy jump can be traced in real time in laboratory experiments and 3D hydrodynamic computations. Theoretical studies of stellar evolution are carried out by computing sequences of stellar models in hydrostatic equilibrium for discrete times separated by fairly large time steps. The buoyancy jump $\triangle b\left(X_{c}, \triangle t\right)$ is then determined by the characteristics of the stellar model, and depends on the time step. Let $d\left(X_{c}\right)$ and $d_{q}\left(X_{c}\right)$ be computed for different time steps $\Delta t$ and $\triangle t / q$. The distance between the boundaries of the regions of convective instability and convective turbulence does not depend on the time step (i.e., $d\left(X_{c}\right)=d_{q}\left(X_{c}\right)$ ) if

$$
\left[\frac{\triangle b\left(X_{c}, \triangle t\right)}{\triangle b\left(X_{c}, \triangle t / q\right)}\right]^{n}=q .
$$

For example, if $q=2$, the ratio $\triangle b\left(X_{c}, \triangle t\right) /$ $\triangle b\left(X_{c}, \triangle t / q\right)$ does not depend on the time step during the entire time for the evolution of the star along the main sequence if the time step is bounded from 

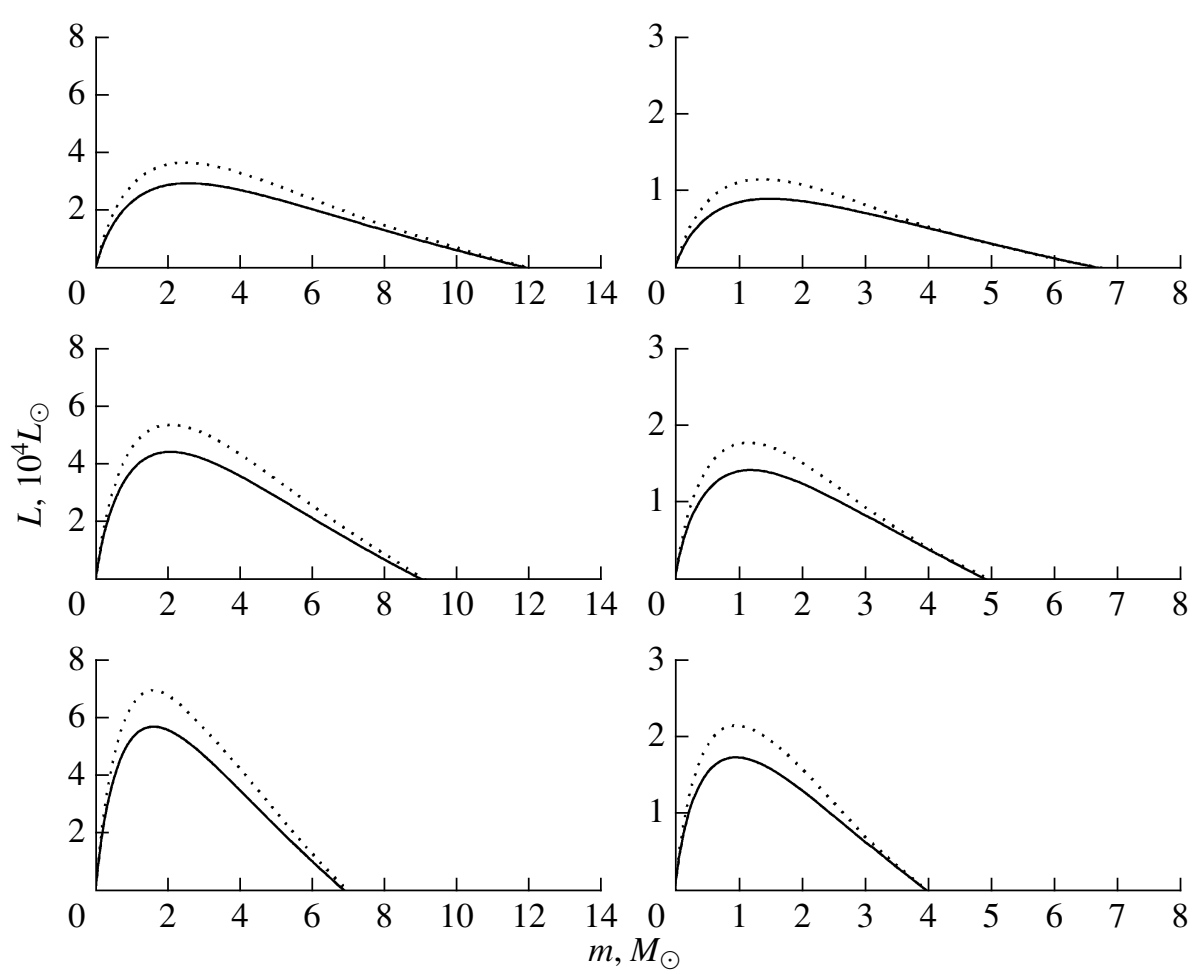

Fig. 1. Convective luminosity in the cores of $24 M_{\odot}$ (left) and $16 M_{\odot}$ (right) stars with hydrogen abundances in the zone of convective turbulence $X_{c}=0.7$ (upper row), $X_{c}=0.4$ (middle row), and $X_{c}=0.1$ (lower row), computed using (7) (solid curve) and mixing-length theory (dotted curve).

above, with this limitation being stricter in advanced phases than in the early phases of the main-sequence evolution (Fig. 2). This ratio also does not depend on the index $n$ in the turbulent-entrainment law (1) and on whether or not mixing of matter located between the boundaries of the regions of convective instability and convective turbulence and matter located in the region unstable to convection is taken into account.

The condition (10) is satisfied for $n=1$ only. Computations of the main-sequence evolution of a $24 M_{\odot}$ star for this case were performed with an appropriate upper limit for the time step, depending on the hydrogen abundance in the region of convective turbulence. The parameter $A$ was fitted in order to ensure that $\alpha\left(X_{c}\right)=d\left(X_{c}\right) / H p\left(X_{c}\right)$ was equal to 0.10 in the zero-age model; here, $H p\left(X_{c}\right)$ was determined at the boundary of the region unstable to convection. The parameter $\alpha\left(X_{c}\right)$ does not remain constant, and gradually decreases as the star evolves along the main sequence (Fig. 3). By substituting( (1), (2), and (4) into (9), we find for $n=1$

$$
d\left(X_{c}\right)=A \frac{\epsilon_{k}\left(X_{c}\right)}{\triangle b\left(X_{c}\right) / \triangle t} .
$$

The rate of hydrogen depletion in the central region of the star increases as the star evolves, due to the increased luminosity. On the contrary, the region of convective instability in the center of the star decreases (Fig. 1). Therefore, the rate of formation of the buoyancy jump at the boundary of the region unstable to convection $\triangle b\left(X_{c}\right) / \Delta t$ increases with time, as the hydrogen abundance in the zone of convective turbulence decreases (Fig. 4a). The increase of the rate of hydrogen depletion also leads to an increase in the convection velocity (Fig. 4a), and therefore to an increase in the turbulent velocity pulsations $V_{t}$ and the convective luminosity (Fig. 1) with time, as the star evolves. The increase in $V_{t}$ means that the kinetic energy of the convective turbulence that goes into mixing the matter of the turbulent region and adjacent regions that are unstable to convection increases. In the turbulent-entrainment model considered, the typical value of the turbulent velocity pulsations $V_{t}$ is determined by (4). The rate of dissipation of the kinetic energy of the turbulent motions $\epsilon_{k}\left(X_{c}\right)$ is computed directly from the stellar model. Therefore, in this turbulent-entrainment model, the kinetic energy that goes into mixing can be indirectly characterized by the value of $\epsilon_{k}\left(X_{c}\right)$. The rate of dissipation of $\epsilon_{k}\left(X_{c}\right)$ increases as the star evolves, but this increase does not compensate the increase in the rate of growth of the buoyancy jump (Fig. 4a). For this reason, the distance $d\left(X_{c}\right)$ between the boundaries of the regions of convective instability and of convective turbulence 


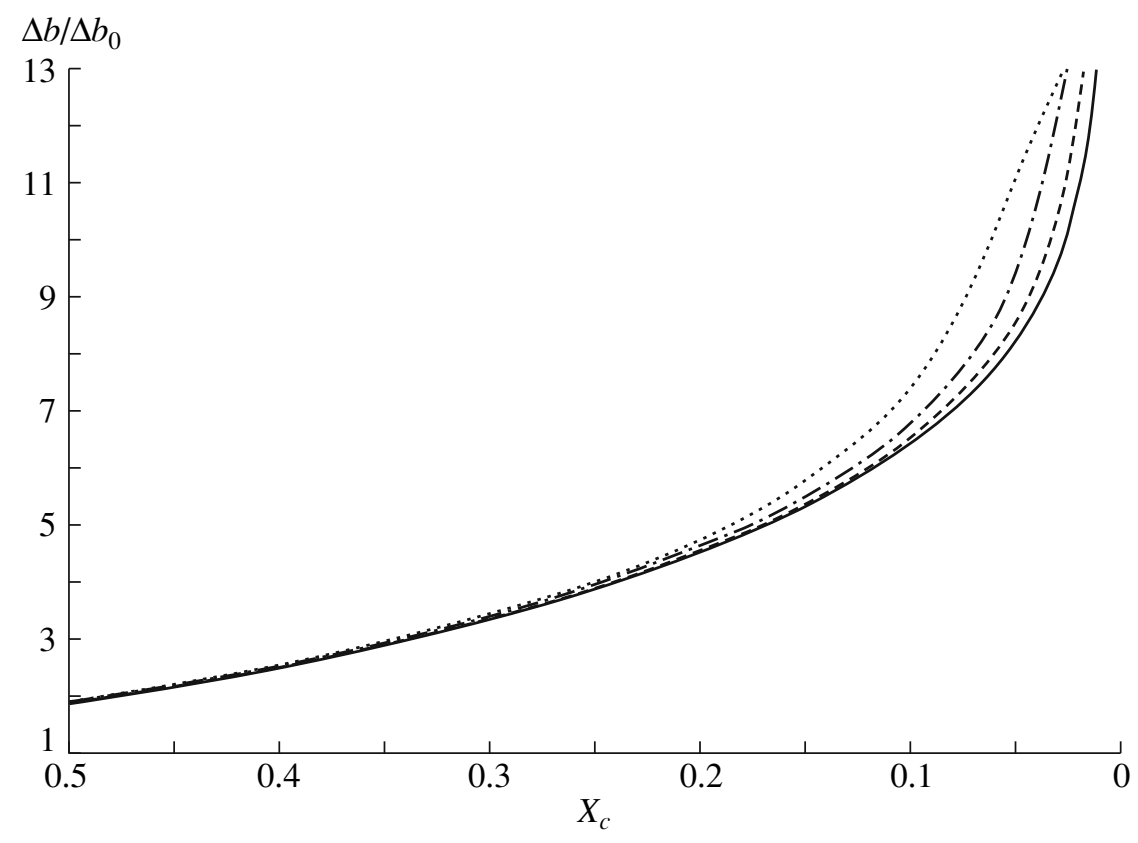

Fig. 2. Time variation of the buoyancy jump $\triangle b$ at the boundary of the region unstable to convection with decreasing hydrogen abundance $X_{c}$ in the region of convective turbulence in a $24 M_{\odot}$ star, with the evolution computed using constant time steps of $2000 \mathrm{yr}$ (solid curve), $4000 \mathrm{yr}$ (dashed curve), $8000 \mathrm{yr}$ (dash-dotted curve), and $16000 \mathrm{yr}$ (dotted curve). All values are normalized to the value for $\triangle b_{0}$ obtained in the first evolutionary model for each time step.

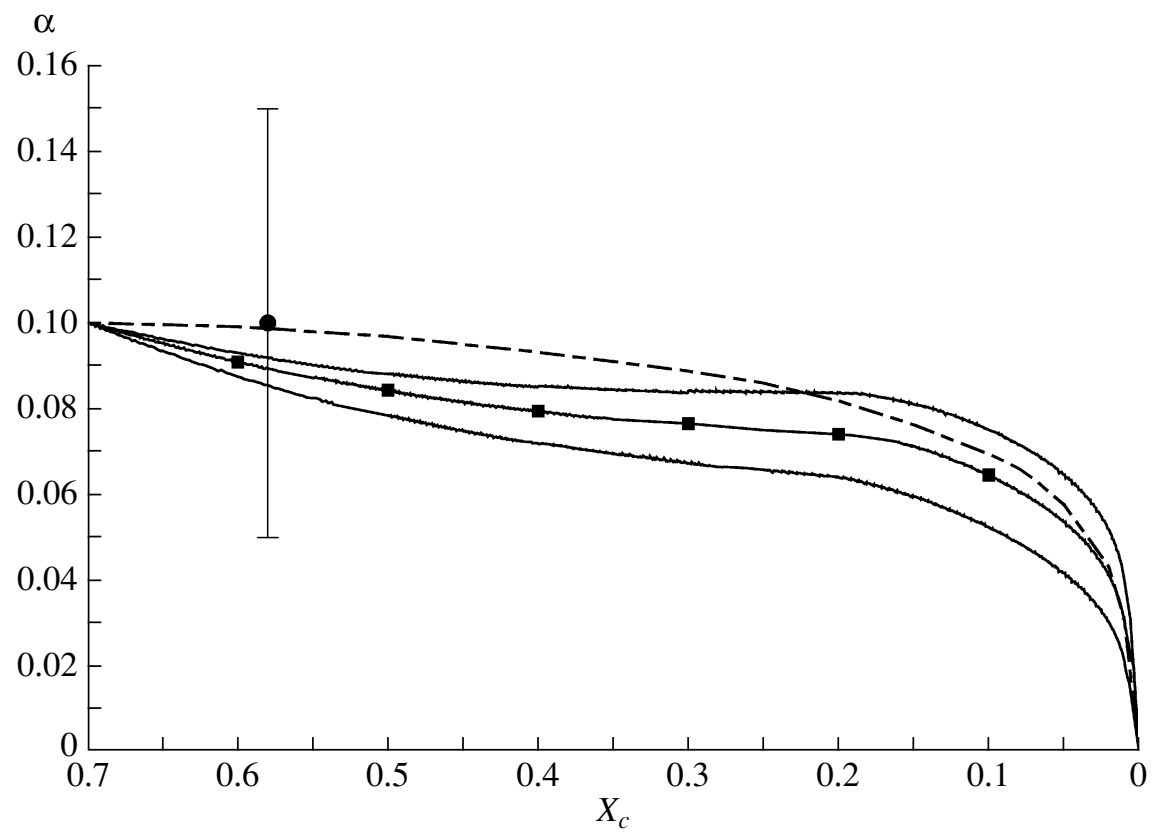

Fig. 3. Dependence of the distance $\alpha$ between the boundaries of the regions of convective instability and convective turbulence at the center of a $24 M_{\odot}$ main-sequence star in units of the pressure scale height on the hydrogen abundance $X_{c}$ in the region of convective turbulence. The dependence computed using the physical turbulent-entrainment model is shown by solid curves for $n=0.85$ (upper), $n=1.00$ (middle), and $n=1.20$ (lower). The squares show the dependence for $n=1.00$, with the convective luminosity computed using mixing-length theory. The dashed curve shows the dependence when the turbulent pulsations of the velocity are equal to the average velocity of convective elements in the boundary convective layer with a thickness $H_{p}$, determined based on mixing-length theory. The average value of $\alpha$ (circle) and the range of its possible values (vertical bar) were determined for the $24 M_{\odot}$ star HD 46202 using asteroseismology methods [4]. 


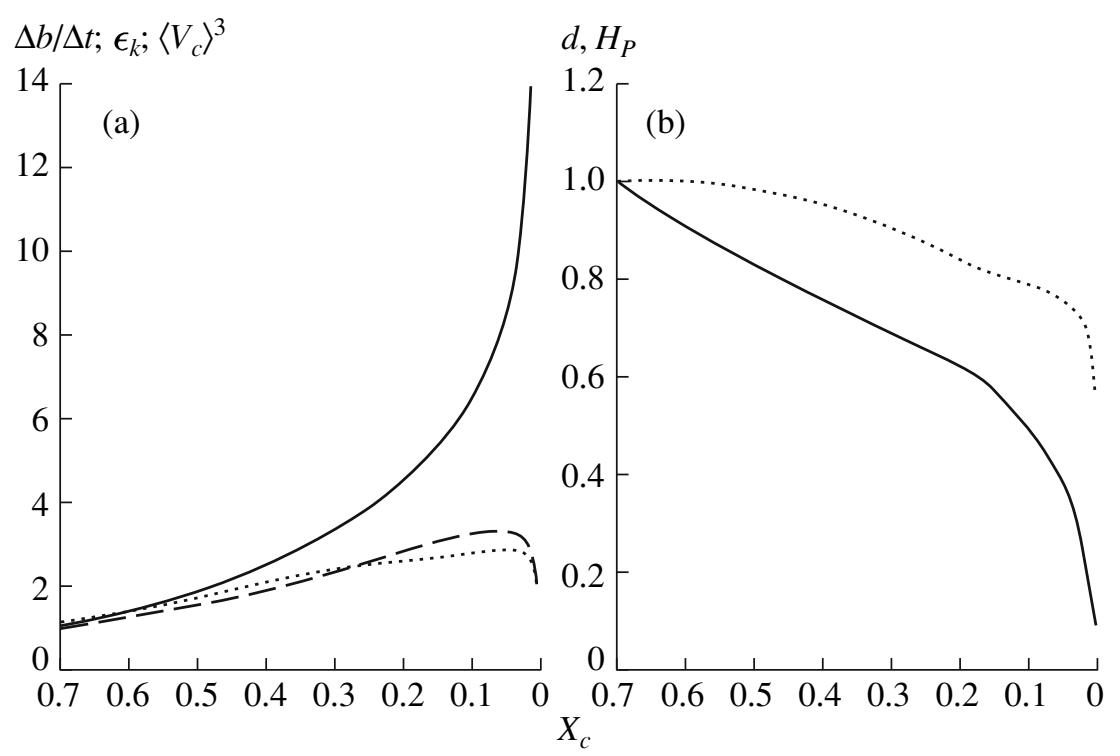

Fig. 4. (a) Velocity of formation of the buoyancy jump (solid) and the rate of dissipation of the kinetic energy of the convective turbulence (dashed) at the boundary of the zone unstable to convection and the cube of the average convective velocity computed using mixing-length theory for the boundary layer with a thickness $H_{p}$ (dotted). (b) Distance $d$ between the boundaries of the regions of convective instability and convective turbulence (solid) and the pressure scale height at the boundary of the region unstable to convection (dotted). These computations were carried out for a $24 M_{\odot}$ star and $n=1.00$. All values are normalized to their zero-age model values.

decreases as the star evolves. This distance should also decrease when $\alpha$ is assumed to be constant, since $H p\left(X_{c}\right)$ also decreases as the star evolves. However, if the energy that goes into mixing is also considered, $d\left(X_{c}\right)$ diminishes more rapidly than $H p\left(X_{c}\right)$ (see Fig. $4 \mathrm{~b})$. Therefore, $\alpha\left(X_{c}\right)$ also decreases with time, as the hydrogen abundance in the region of convective turbulence decreases.

The seismological parameters of the stars were derived using models computed assuming that $\alpha$ remains constant during the star's evolution along the main sequence. Most of the stars studied are in advanced stages of their main-sequence evolution and have reduced central hydrogen abundances $X_{c} \leq$ 0.35 (Table 1 ). Since $\alpha\left(X_{c}\right)$ varies during the star's evolution, the values $\alpha$ obtained for these stars cannot be used to determine $A$. Unfortunately, $\alpha_{0}$ has been determined for only one weakly evolved starHD46202. The mass measured from seismology data and the age of this star are $24 M_{\odot}$ and 2 Myr [4]. The hydrogen abundance in the convective turbulence zone of a star of this mass and age is $\sim 0.58$. The chosen value of $A$ was based on the seismological value of $\alpha_{0}$ for this star (Fig. 3).

The evolution of one $24 M_{\odot}$ star was studied for the case when, in the calculation of the rate of dissipation of the kinetic energy of the turbulent motion $\epsilon_{k}\left(X_{c}\right)$, the convective luminosity was calculated based on mixing-length theory rather than (7). In this case, the value of $\epsilon_{k}\left(X_{c}\right)$ was $\sim 20 \%$ larger than when (7) was used during the entire main-sequence stage. The parameter $A$ was fit such that the zero-age model had $\alpha_{0}=0.10$, and it turned out to be $\sim 20 \%$ lower than when (7) was used. The computed dependence $\alpha\left(X_{c}\right)$ essentially does not differ (Fig. 3) from the one obtained when (7) was used to determine $\epsilon_{k}\left(X_{c}\right)$.

The typical value of the turbulent velocity pulsations $V_{t}$ at the boundary of the region of convective turbulence is comparable to the radial component of the velocity of the convective flow [13]. The evolution of one $24 M_{\odot}$ star was studied assuming that $V_{t}$ was equal to the average convective velocity derived using mixing-length theory, for a layer with a thickness of $H_{p}$ adjacent to the boundary of the region unstable to convection. At the beginning of the main-sequence evolution, this average value is $\sim 2 \times 10^{4} \mathrm{~cm} / \mathrm{s}$. The radial component of the convective velocity at the boundary of the region unstable to convection found in [13] is an order of magnitude higher $\left(\sim 2 \times 10^{5} \mathrm{~cm} / \mathrm{s}\right)$, since the luminosity at the bottom of the computational domain was artificially enhanced. It was not necessary to compute the rate of dissipation of the kinetic energy of the turbulent motions $\epsilon_{k}\left(X_{c}\right)$ for the stellar evolution considered here. The velocity $V_{e}$ can be found from (1), (2), and (3). In this case, the parameter $\alpha\left(X_{c}\right)$ also decreases as the star evolves (Fig. 3), since the increase in the convective velocity does not balance the increase in the rate of formation of the buoyancy jump (Fig. 4a). The turbulent pulsations of the velocity determined 
using (4) comprise $\sim 1.6 \times 10^{4} \mathrm{~cm} / \mathrm{s}$ at the beginning of the star's evolution along the main sequence, and do not exceed the average convective velocity during the entire main-sequence stage. In the case considered, the parameter $\alpha\left(X_{c}\right)$ remains larger than when the typical velocity of the turbulent motions is derived using (4).

If $n \neq 1$, condition (10) is not satisfied. However, since the ratio $\triangle b\left(X_{c}, \triangle t\right) / \triangle b\left(X_{c}, \triangle t / q\right)$ does not depend on the time step for small enough $\triangle t$ for such values of $n$, it is possible to compute $\alpha\left(X_{c}\right)$, which does not depend on the choice of time step (Fig. 3). This requires that the stellar evolution be compuated with a constant time step, and the parameter $A$ must be adjusted at every time step so as to satisfy the requirement that $\alpha_{0}$ has the chosen value in the zeroage model. If condition (10) is not satisfied for $n \neq 1$, $A$ becomes a formal fitting parameter.

Turbulent entrainment at the boundary of the main-sequence convective core changes the profile of the hydrogen distribution in the zone of variable chemical composition, resulting in an increase of the mass of synthesized helium and an increase in the main-sequence lifetime compared to the standard case. For comparison, we computed the evolution of a $24 M_{\odot}$ star with constant values $\alpha_{c}=0.05$ and $\alpha_{c}=0.10$. The hydrogen profile that forms during the evolution beyond the boundary of the region of convective instability depends on the index $n$ in the turbulent-entrainment law, and is flatter than in the standard case and in the case of constant $\alpha_{c}$ (Fig. 5). With fixed $\alpha_{0}$, the increase of the mass of hydrogen burnt to form helium and of the stellar lifetime also depend on $n$ (Table 2 ).

The seismological values of $\alpha$ for the stars studied display a large scatter (Table 1). Further, the possible values of $\alpha$ for stars with close masses do not overlap (for instance, in the pairs $\beta$ CMa and HD 180642 or $\delta$ Cet and $\nu$ Eri). The mass of the convective core of the main-sequence star may also increase compared to the standard case due to the influx of matter from its radiative envelope because of shear mixing due to rotation [24]. The flux of hydrogen from the radiative turbulent envelope into the convective core has its maximum in the second half of the star's evolution along the main sequence [25]. The intensity of hydrodynamic transport processes in the non-convective layers of a star is determined by the momentum and Schmidt number in the turbulent radiative envelope $[25,26]$, and can be different for stars of the same mass. For example, the mass of the chemically homogeneous region in the central part of the star during the second half of its main-sequence evolution is determined by the combined action of turbulent entrainment and shear turbulence, and can be different for stars of the same mass. If stellar
Table 2. Increase in the mass of synthesized helium and the main-sequence lifetime for a $24 M_{\odot}$ star in the physical turbulent-entrainment model with $\alpha_{0}=0.10$ and in the model with $\alpha_{c}=$ constant

\begin{tabular}{l|c|c|c|c|c}
\hline \multirow{2}{*}{ Value } & \multirow{2}{*}{$\alpha_{c}=0.05$} & \multicolumn{3}{|c|}{$\alpha_{0}=0.10$} & \multirow{2}{*}{$\alpha_{c}=0.10$} \\
\cline { 3 - 5 } & & $n=1.20$ & $n=1.00$ & $n=0.85$ & \\
\hline$\Delta M$ & $4.5 \%$ & $7.0 \%$ & $7.5 \%$ & $7.9 \%$ & $9.1 \%$ \\
$\Delta t$ & $2.6 \%$ & $3.6 \%$ & $4.0 \%$ & $4.3 \%$ & $5.0 \%$ \\
\hline
\end{tabular}

models in which additional mixing is accounted for by specifying the time-invariant parameter $\alpha$ are used to analyze the frequency spectrum, this can result in different values of the latter parameter for stars of similar mass.

The presence in the stellar interiors of a layer with variable chemical composition located above the region of convective turbulence results in specific features in the periods of the $\mathrm{g}$ modes of high order and low degree. The deviations of the differences of the pulsation periods for neighboring orders from the average value vary according to a sinusoidal law. If the hydrogen profile in the layer of variable chemical composition is computed for a model with constant $\alpha$, the amplitude of the sinusoid is constant, while the amplitude decreases with increasing order of the mode when the profile is determined by turbulent diffusion [27, 28]. Such an amplitude decline was detected for HD 50230 [12]. The difference in the hydrogen profiles that are formed in the zone of variable chemical composition if the two processes described above are considered separately is also reflected in the pulsation periods for low-order $\mathrm{p}$ and $\mathrm{g}$ modes [29]. However, precisely these modes were used to determine the parameter $\alpha$ for most stars from Table 1 . For the flatter hydrogen distribution obtained in the zone of variable chemical composition when a physical model of turbulent entrainment is applied, the amplitude of the variability of the deviations of the differences between the periods of the gravitational pulsation modes of sequential orders from the average value may be smaller than in the case of constant $\alpha$, for the same central abundance. This variability amplitude serves as an indicator of the stellar age in asteroseismology. Therefore, including turbulent entrainment should influence the seismological parameters of the stars.

The seismological values of $\alpha$ for the studied stars with the highest central hydrogen abundances (HD 46202 and HD 129929) are 0.05-0.15 (Table 1). Having in mind these values, we computed the evolution of a $16 M_{\odot}$ star using the turbulent-entrainment model with $\alpha_{0}=0.15$ and $n=0.85,1.00$, and 1.20 , as well as for the cases of constant $\alpha_{c}=0.10$ and 


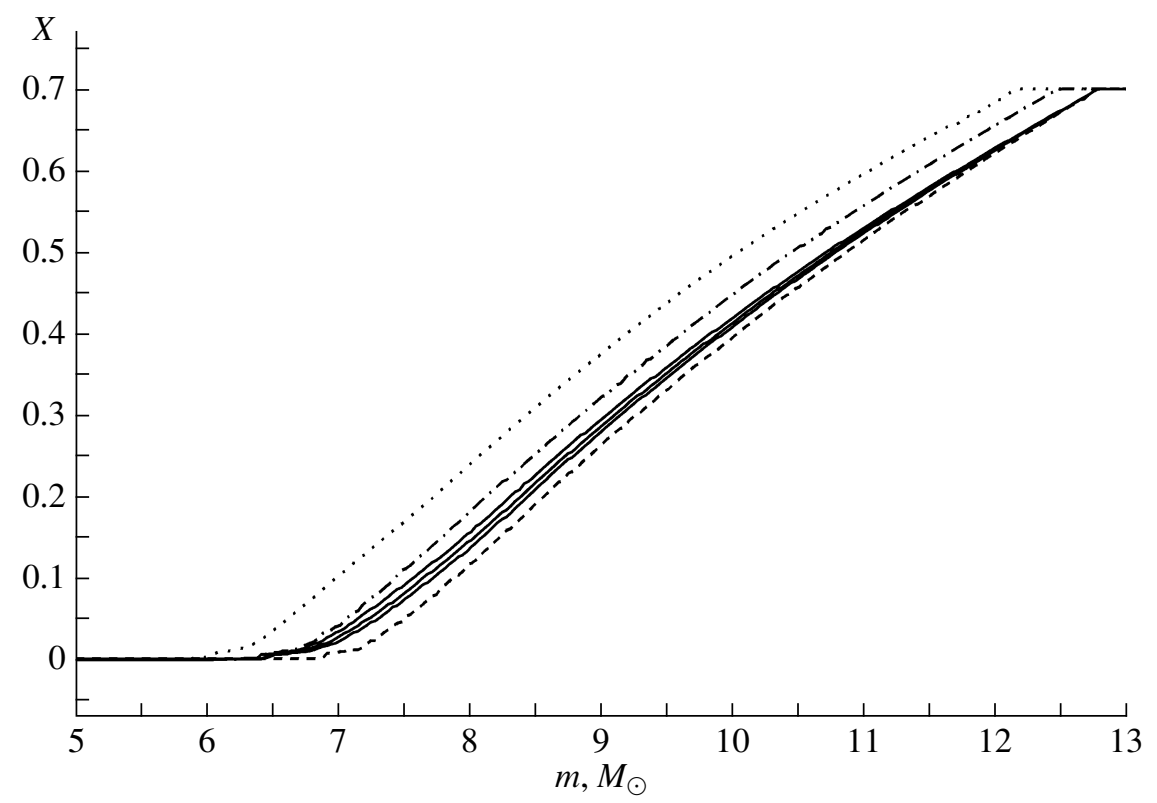

Fig. 5. Hydrogen abundance $X$ at the center of a $24 M_{\odot}$ star. Solid curves correspond to the case when the boundary of the convective turbulence region was determined using the turbulent-entrainment model, with $n=1.20$ (upper), $n=1.00$ (middle), and $n=0.85$ (lower). The dashed and dot-dashed curve show the case of constant $\alpha_{c}$ equal to 0.10 and 0.05 , respectively. The dotted curve shows the case when mixing occurs in the region of convective instability only.

$\alpha_{c}=0.15$. In the model with turbulent entrainment, the increase in the mass of hydrogen converted into helium during the main-sequence lifetime is lower that for the model with constant $\alpha_{c}=0.15$ (Table. 3), which is necessary to bring the computed and observed increases in the luminosity during this evolutionary stage into agreement. However, the mass of synthesized helium also increases due to shear turbulence. For example, hydrogen from the radiative envelope is transported into the convective core quite intensively in the case of the fast rotation typical for Be stars [25]. Thus, if the turbulent Schmidt number is $0.7-0.4$, the mass of helium synthesized during the main-sequence lifetime of a $16 M_{\odot}$ star can increase by $25-50 \%$. It is possible that hydrogen from the radiative turbulent envelope is also transferred into the zone of convective turbulence

Table 3. Increase in the mass of synthesized helium and the main-sequence lifetime for a $16 M_{\odot}$ star in the physical turbulent-entrainment model with $\alpha_{0}=0.15$ and in the model with $\alpha_{c}=$ constant

\begin{tabular}{l|r|r|r|r|l}
\hline \multirow{2}{*}{ Value } & \multirow{2}{*}{$\alpha_{c}=0.10$} & \multicolumn{3}{|c|}{$\alpha_{0}=0.15$} & \multirow{2}{*}{$\alpha_{c}=0.15$} \\
\cline { 3 - 5 } & & $n=1.20$ & $n=1.00$ & $n=0.85$ & \\
\hline$\Delta M$ & $14.2 \%$ & $16.5 \%$ & $19.0 \%$ & $20.8 \%$ & $23.6 \%$ \\
$\Delta t$ & $7.9 \%$ & $8.9 \%$ & $10.1 \%$ & $11.2 \%$ & $12.5 \%$ \\
\hline
\end{tabular}

in the case of lower rotation rates, but with a lower intensity.

\section{CONCLUSION}

The distance $\alpha\left(X_{c}\right)$ between the boundaries of the regions of convective instability and convective turbulence derived according to the physical model for turbulent entrainment [13-15] (expressed in the units of the pressure scale height, as is usual in the astrophysical literature) is not constant, and decreases as 16 and $24 M_{\odot}$ stars evolve along the main sequence. The exact behavior of $\alpha\left(X_{c}\right)$ is determined by the fraction of the kinetic energy of the convective turbulence that can go into mixing the matter of the region of convective turbulence and the overlying layers that are stable against convection. In the turbulent-entrainment model considered, this fraction is measured using the rate of dissipation of the kinetic energy of the turbulent flow $\epsilon_{k}\left(X_{c}\right)$, which can be computed directly using a stellar model. A method for determining $\epsilon_{k}\left(X_{c}\right)$ was proposed in [14] for the case of convective turbulence in a carbonburning shell in a $23 M_{\odot}$ star. Confident application of the turbulent-entrainment model in computations of stellar structure and evolution requires confirmation of the correctness of the derivation of $\epsilon_{k}\left(X_{c}\right)$ using this method in other stages of stellar evolution, including the main sequence stage. A crude determination of $\alpha\left(X_{c}\right)$, when the convective velocity obtained 
from mixing-length theory is used for the typical values of the turbulent velocity pulsations, results in qualitatively the same temporal behavior for $\alpha\left(X_{c}\right)$. If $\alpha_{0}$ is fixed at the beginning of a star's evolution along the main sequence, the behavior of $\alpha\left(X_{c}\right)$ does not depend on whether the convective luminosity is derived as in [14] or based on mixing-length theory.

Since $\alpha\left(X_{c}\right)$ decreases in the course of a star's evolution, the distribution of the hydrogen abundance by mass in the layer of variable chemical composition is flatter than in standard models of stellar evolution and in models where $\alpha$ is specified to be constant. A flatter hydrogen-abundance distribution could influence the pulsation frequencies of the stars, and accordingly their derived seismological parameters.

In our computations of the evolution of mainsequence stars, the parameter $A$ in the turbulententrainment law was ajusted so that $\alpha_{0}$ acquired a prespecified value. For $n=1$ and $\alpha_{0}=0.1, A=$ $4.425 \times 10^{-4}$ and $4.054 \times 10^{-4}$ for the 16 and $24 M_{\odot}$ model stars, respectively. The parameter $A$ contains an uncertainty, due to the possible difference of the largest scale of the turbulence in the expression for the bulk Richardson number (2) and in the expression for the rate of dissipation of the kinetic energy of the turbulent flow (4). After removing this uncertainty using the values of these variables for a carbonburning shell [13, 15], A still remains much lower than the value obtained in [13]. Computing the stellar evolution using $A$ (instead of $\alpha_{0}$ ) requires that the source of this disagreement be found. It is possible that the artificial enhancement of the luminosity at the bottom of the computational domain, which is necessary for $3 \mathrm{D}$ computations of the turbulent entrainment, influenced the value of $A$. It is possible also that the largest turbulence scales in (2) and (4) are different in the convective core and convective shell. If $\alpha_{0}$ is constant, the increase in the mass of helium synthesized on the main sequence is larger for lower-mass stars. If $A$ is constant, $\alpha_{0}$ decreases with the mass of the star. This may be reflected in the dependence of the additional mass of synthesized helium on the mass of the star. If the convective luminosity is derived using mixing-length theory, the value of $A$ is $\sim 20 \%$ lower, for the same $\alpha_{0}$. Hence, if a fixed value of $A$ is used, $\alpha\left(X_{c}\right)$ will depend on the details of the turbulent-entrainment model.

In the early stages of main-sequence stellar evolution, shear turbulence and meridional circulation due to the star's rotation do not result in appreciable mixing of the matter outside the region of convective turbulence in the center of the star, even for large rotational velocities [25]. Turbulent entrainment is the only mixing mechanism that may change the stellar structure in this evolutionary stage, compared to standard models. The hydrogen profile in the zone of variable chemical composition that forms above the region of convective turbulence in the early stages of stellar evolution does not depend on the index $n$ in the turbulent-entrainment law. Therefore, another possible way to obtain information about $A$ is determining the parameter $\alpha_{0}$ using asteroseismology, for stars that are in the initial stages of their main-sequence evolution. The theoretically computed lower boundary of the instability strip for slowly pulsating B stars with $3 \leq M / M_{\odot} \leq 7$ in the Hertzsprung-Russell diagram practically overlaps with the zero-age main sequence, while it is close to the zero-age main sequence for $\beta$ Cep pulsating stars with $7 \leq M / M_{\odot} \leq$ $20[30,31]$. Currently, $\alpha_{0}$ has been determined only for the star HD 46202 , which has a mass of $24 M_{\odot}$ and a central hydrogen abundance $X_{c} \sim 0.58$; the problem of the excitation of the observed pulsation modes of this star remains theoretically unsolved.

To explain the observed increase in the luminosities of main-sequence stars, the mass of helium synthesized in hydrogen-burning nuclear reactions must be higher than in standard stellar models by the amount obtained in computations with constant $\alpha_{c} \sim 0.15-0.17$ [1]. Given that, according to the turbulent-entrainment model, $\alpha\left(X_{c}\right)$ decreases with time, and guided by the value of $\alpha_{0}$ for HD 46202 , we find that turbulent entrainment alone is not sufficient to provide the required increase in the mass of synthesized helium. Another possible way to enhance this mass is to transport hydrogen from the radiative envelope involved in shear turbulence into the region of convective turbulence [25].

Determining the relative roles of various mixing processes acting in stellar interiors requires improvement of the turbulent-entrainment model; i.e., determining whether the expression for the rate of dissipation of the kinetic energy of the turbulent motion is valid for different stages of stellar evolution, finding values of $A$ for stars of different masses, and finding the value of $\alpha_{0}$ using asteroseismology methods applied to a statistically significant sample of stars.

\section{ACKNOWLEDGMENTS}

This study was partially supported by the Federal Targeted Program "Research and Development in Priority Directions of the Development of the Scientific and Technological Complex of Russia" for 20072013 (state contract No. 14.518.11.7064).

\section{REFERENCES}

1. A. Maeder and J.-C. Mermilliod, Astron. Astrophys. 93, 136 (1981).

2. G. Meylan and A. Maeder, Astron. Astrophys. 108, 148 (1982). 
3. J.-C. Mermilliod and A. Maeder, Astron. Astrophys. 158, 45 (1986).

4. M. Briquet, C. Aerts, A. Baglin, et al., Astron. Astrophys. 527, $112(2011)$.

5. A. Mazumdar, M. Briquet, M. Desmet and C. Aerts, Astron. Astrophys. 459, 589 (2006).

6. C. Aerts, M. Briquet, P. Degroote, et al., Astron. Astrophys. 534, 98 (2011).

7. C. Aerts, S. V. Marchenko, J. M. Matthews, et al., Astrophys. J. 642, 470 (2006).

8. M. Briquet, T. Morel, A. Thoul, et al., Mon. Not. R. Astron. Soc. 381, 1482 (2007).

9. M. Desmet, M. Briquet, A. Thoul, et al., Mon. Not. R. Astron. Soc. 396, 1460 (2009).

10. A. A. Pamyatnykh, G. Handler, and W. A. Dziembowski, Mon. Not. R. Astron. Soc. 350, 1022 (2004).

11. M.-A. Dupret, A. Thoul, R. Scuflaire, et al., Astron. Astrophys. 415, 251 (2004).

12. P. Degroote, C. Aerts, A. Baglin, et al., Nature 464, $259(2010)$.

13. C. A. Meakin and D. Arnett, Astrophys. J. 667, 448 (2007).

14. C. A. Meakin and D. Arnett, Astrophys. Space Sci. 328,221 (2010).

15. D. Arnett, C. A. Meakin, and P. A. Young, Astrophys. J. 690, 1715 (2009).

16. P. F. Linden, J. Fluid Mech. 71, 385 (1975).
17. L. D. Landau and E. M. Lifshitz, Course of Theoretical Physics, Vol. 6: Fluid Mechanics (Nauka, Moscow, 1986; Pergamon, New York, 1987).

18. B. Paczynski, Acta Astron. 20, 47 (1970).

19. E. I. Staritsin, Astron. Rep. 43, 592 (1999).

20. C. A. Iglesias and F. J. Rogers, Astrophys. J. 464, 943 (1996).

21. D. R. Alexander and J. Ferguson, Astrophys. J. 437, 879 (1994).

22. H. J. S. Fernando, Ann. Rev. Fluid Mech. 23, 455 (1991).

23. E. Bohm-Vitenze, Z. Astrophys. 46, 108 (1958).

24. E. I. Staritsin, Astron. Rep. 45, 467 (2001).

25. E. I. Staritsin, Astron. Lett. 35, 413 (2009).

26. E. I. Staritsin, Astron. Lett. 33, 93 (2007).

27. A. Miglio, J. Montalban, P. Eggenberger, and A. Noels, Astron. Nachr. 329, 529 (2008).

28. A. Miglio, J. Montalban, A. Noels, and P. Eggenberger, Mon. Not. R. Astron. Soc. 386, 1487 (2008).

29. J. Montalban, A. Miglio, P. Eggenberger, and A. Noels, Astron. Nachr. 329, 535 (2008).

30. A. Miglio, J. Montalban, and M.-A. Dupret, Mon. Not. R. Astron. Soc. 375, L21 (2007).

31. T. Zdravkov and A. A. Pamyatnykh, J. Phys. Conf. Ser. 118, 012079 (2008).

Translated by L. Yungel'son 